\title{
Leukocyte count on admission as a predictor of clinical outcome in the expectant management for women with preterm prelabor rupture of membranes
}

\author{
Deeksha Pandey ${ }^{1}$, Saurav Bhagat ${ }^{1}$, Vidhi Vanya ${ }^{1}$, V S Binu ${ }^{1}$, Muralidhar Pai ${ }^{1}$, Pratap Kumar \\ Sri Lanka Journal of Obstetrics and Gynaecology 2011; 33: 51-55
}

\begin{abstract}
Objective: We hypothesized that a single value of leukocyte count, on admission might be a helpful predictor to prognosticate the clinical outcome and plan the management (early delivery versus conservative management) in patients presenting with pre term pre-labour rupture of membranes (PPROM).
\end{abstract}

Design: Observational study.

Setting: This study was conducted in a tertiary care centre.

Population or sample: 127 pregnancies complicated with PPROM.

Method: A novel scoring system was devised depending upon the final clinical performance.

Main outcome measure: Adverse fetal, neonatal and maternal outcome.

Result: Based on the score cut off for the total leukocyte count was calculated to prognosticate the outcome. Leukocyte count of $15,850 / \mathrm{mm}^{3}$, at the time of admission was found to be able to predict the clinical outcome with a sensitivity of $85.7 \%$ and specificity of $87.6 \%$, in a case of PPROM. Whereas the individual components of differential leukocyte counts were found to be not of much help in this regard.

Conclusion: Leukocyte count of $15,850 / \mathrm{mm}^{3}$ at admission can be used to prognosticate outcome in pregnancies complicated with PPROM.

Key words: preterm prelabor rupture of membranes, total leukocyte count, differential count, prematurity, infectious morbidity.

\footnotetext{
${ }^{1}$ Department of Obstetrics and Gynaecology, Kasturba Hospital, Manipal, India.

Correspondence: Deeksha Pandey

Email:deekshiiiobg@gmail.com
}

\section{Introduction}

Preterm prelabor rupture of the membranes (PPROM) refers to spontaneous rupture of membranes in the absence of labor pains, before 37 completed weeks of gestation. On an average PPROM complicates $2-4.5 \%$ of pregnancies, globally ${ }^{1-3}$. Diagnosis of PPROM is considered one of the most tragic obstetrical events. Associated problems include prematurity in early delivery versus infectious morbidity for both mother and fetus with conservative management to prolong the pregnancy. Simultaneously the fact to be kept in mind is that the majority of conservatively managed women will deliver within one week and do not accrue the benefits of extended pregnancy prolongation ${ }^{4}$. However, this small prolongation of pregnancy might be of great benefit in order to administer steroid prophylaxis. This will help to reduce the incidence of respiratory distress syndrome (RDS), one of the most dreaded complications of prematurity. RDS occurs in $10-40 \%$ of women with PPROM and is responsible for approximately $40-70 \%$ of neonatal deaths ${ }^{5}$. Moreover a single course of antenatal steroids has also been shown to substantially reduce the incidence of intraventricular hemorrhage (IVH) and necrotizing enterocolitis (NEC) in preterm neonates ${ }^{6}$.

The delicate balance between the benefits of little added fetal maturity while avoiding the potential harms of retaining the fetus in-utero mandates careful vigilance and anticipation. The important issues to be addressed with utmost care, during this approach of management are predicting the outcome and counseling the parents about the prognosis of the newborn. Amniotic fluid analysis by means of amniocentesis has been advocated in some centers to prognosticate fetal and maternal outcome in cases complicated with PPROM ${ }^{7}$. The invasive character of amniocentesis as well as difficulty of this procedure in presence of oligohydramnios are its major limitations. Maternal blood markers like leukocyte count, C reactive protein (CRP), erythrocyte sedimentation ratio (ESR) and interleukin 6 (IL-6) have also been studied in this context, to predict subclinical infection in cases of PPROM ${ }^{8,9}$. In a recent metaanalysis CRP was found to be moderately predictive of histological chorioamnionitis in cases of PPROM ${ }^{10}$. Disappointing sensitivities and positive predictive values however have kept these tests far away from 
evidence based clinical application till date. All these studies however, have tried to correlate maternal serum markers either with positive amniotic fluid cultures or with histologic chorioamnionitis. In our study we hypothesized that a single value of leukocyte count, on admission might be a helpful predictor to prognosticate clinical outcome and plan management (early delivery versus conservative management) in cases of PPROM.

\section{Material and methods}

This observational study was conducted in a tertiary care centre, in 127 pregnancies complicated with PPROM. This study was conducted at Kasturba Hospital, Manipal, India, during a period of 4 years and 10 months from January 2006 - October 2010. All patients included in this study presented with complaints of leaking per vagina during 24-37 completed weeks period of gestation. As per our management policy, after the diagnosis of PPROM was confirmed by visualizing a clear leak on sterile speculum examination, patient was admitted to hospital. Gestational age was confirmed and fetal well being documented. PPROM beyond 37 completed weeks of gestation, multiple pregnancies, and cases with evidence of chorioamnioninitis at the time of admission were excluded from the study. Initial leukocyte count (total and differential) was performed and parenteral antibiotics started. Cases falling in the category of 34-37 weeks period of gestation were induced if not contraindicated. Steroid prophylaxis (two doses of injection betamethasone, intramuscular $12 \mathrm{mg}$ each, 24 hours apart) was administered to those less than 34 weeks period of gestation. These cases were followed up with daily total leukocyte count, and alternate day amniotic fluid index (AFI). Delivery was planned once the gestation crossed 34 weeks, leukocyte count showed an increasing trend, AFI showed a decreasing trend or was found to be below $5 \mathrm{~cm}$, or if there was spontaneous onset of labor. During expectant management cord prolapse, umbilical cord compression, or placental abruption mandating urgent delivery were also looked for.

Immediately after delivery all newborns were attended by the neonatologist. If required, they were admitted to the neonatal intensive care unit (NICU) for further management. These infants were observed for complications related to prematurity (RDS, NE, IVH) and infectious morbidities (sepsis). Duration of NICU stay, condition on discharge, and neonatal death was also recorded. Clinically evident chorioamnionitis, primary or secondary postpartum hemorrhage (PPH), and endometritis are the maternal complications included in the study.

After collecting the data obtained from 127 pregnancies complicated with PPROM and selected for the study, a scoring system was devised for maternal and fetal complications. Scores was attributed according to the graveness of the variables on the final fetomaternal outcome. Scoring system is tabulated in Table 1. On adding up all these numbers a final score was given to every patient. A cutoff value of 8 was chosen, as in all instances if the score was more than or equal to 8 , the complications were unaccepatable as either they were too many or too bad. Using this cutoff ( $\geq 8$ serious complications and $<8$ acceptable morbidities), receiver operating characteristic (ROC) curves were generated first with the total leucocyte count (TLC) and then with the various components of differential leukocyte count (DLC). The data were entered and analyzed in SPSS 15 (SPSS Inc; Chicago, IL) and Student's t test was used for comparing continuous variables while Fisher's exact test for categorical variables. $P$ value less than 0.05 was considered as significant.

Table 1. A new scoring system to quantify the overall maternal and fetal clinical outcome in cases of PPROM

\begin{tabular}{|c|c|c|c|}
\hline Fetal variables & Score & Maternal variables & Score \\
\hline Respiratory distress syndrome (RDS) & 3 & Chorioamnionitis & 2 \\
\hline Neonatal sepsis & 3 & Postpartum hemorrhage & 2 \\
\hline Intraventricular hemorrhage (IVH) & 3 & Endometritis & 1 \\
\hline Necrotizing enterocolitis (NEC) & 3 & & \\
\hline Cesarean delivery & 1 & \multirow{3}{*}{\multicolumn{2}{|c|}{$\begin{array}{l}\text { Cut off } 8 \\
\text { A. Score }<8 \text { means in any case, the combination } \\
\text { of - at the maximum } 2 \text { major (though } \\
\text { treatable) complications }\end{array}$}} \\
\hline Intrauterine death (IUD) & 5 & & \\
\hline Neonatal death & 5 & & \\
\hline Prolonged NICU stay (beyond 2 weeks) & 2 & \multicolumn{2}{|c|}{$\begin{array}{l}\text { B. Score } \geq 8 \text { means either } 3 \text { or more serious } \\
\text { complications, or mortality because of the } \\
\text { complication of PPROM }\end{array}$} \\
\hline
\end{tabular}




\section{Results}

The mean age of women in our study population was $27.42 \pm 4.06$ years, and $75.5 \%$ of those were primigravida. Mean period of gestation at the time of rupture of membranes was around $34.1 \pm 2.8$ weeks. The mean interval between the time of onset of leaking per vagina and blood withdrawn for leukocyte count was found to be $5 \pm 1.2$ hours.

After scoring the various outcomes as mentioned in Table 1, demographic and clinical characteristics of the two groups: A. Score $<8(n=113)$ and B. Score $\geq 8$ $(n=14)$ were compared and found to be comparable.

\section{(A)Total leukocyte count and clinical outcome}

On plotting the ROC curve with total leukocyte count on admission and the final outcome, as mentioned before, the area under curve was found to be 0.880 suggesting that the test is good (Figure 1 ). It can be inferred from the curve that a cut off value of $15,850 / \mathrm{mm}^{3}$ of TLC gives a sensitivity of $85.7 \%$ and specificity of $87.6 \%$.

\section{ROC Curve}

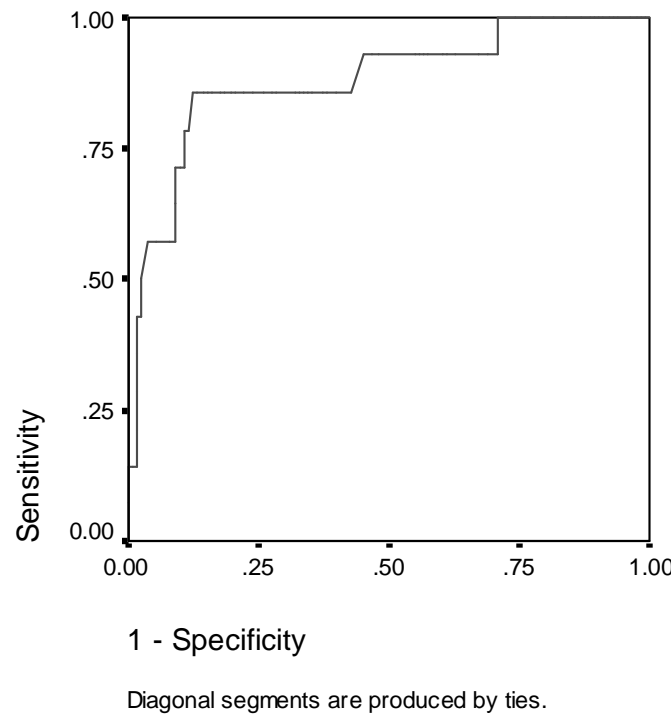

Figure 1. Receiver operating characteristic curve of total leukocyte count in relation with the outcome measures (according to the scoring system in Table 1).

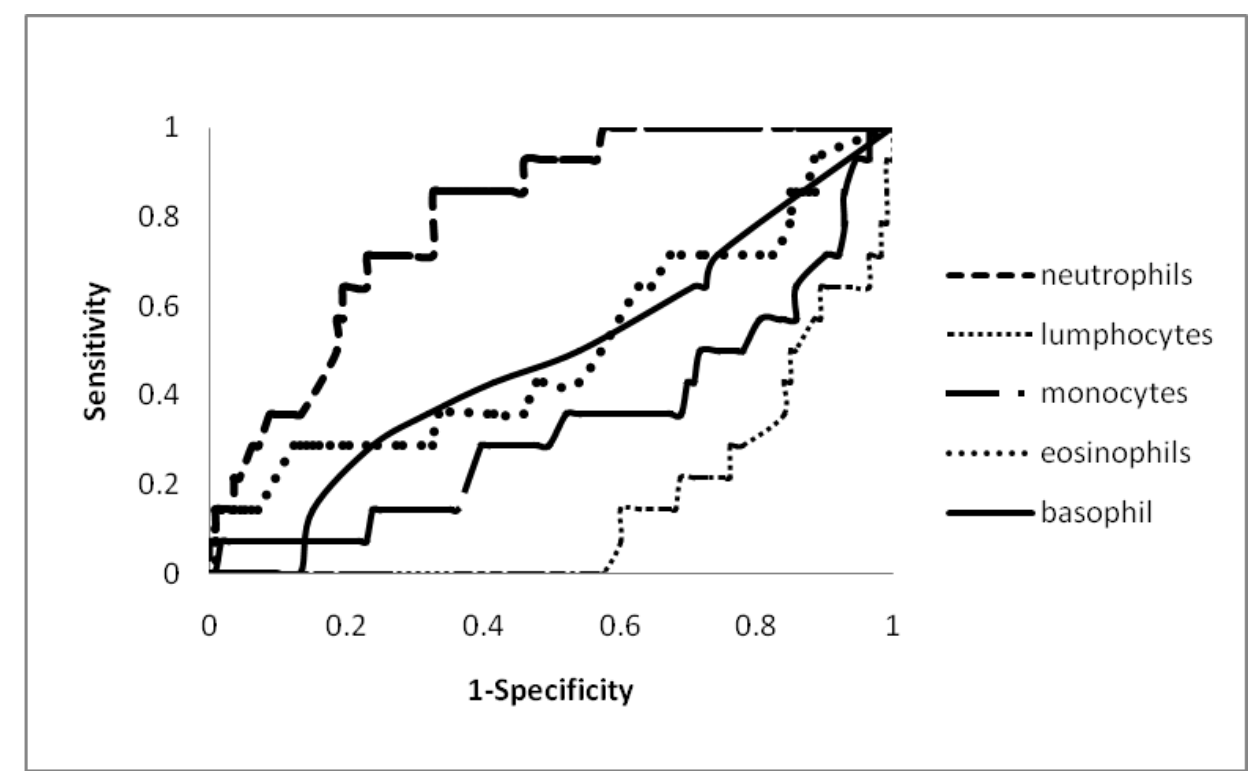

Figure 2. Receiver operating characteristic curve of various components of differential leukocyte count in relation with the outcome measures (according to the scoring system in Table 1 ). 
Table 2. Demographic and clinical characteristics of population studied (age and parity)

\begin{tabular}{llll}
\hline & $\begin{array}{l}\text { A. Score }<8 \\
(n=113)\end{array}$ & $\begin{array}{l}\text { B. Score } \geq 8 \\
(n=14)\end{array}$ & P value \\
\hline Maternal age in years - mean (SD) & $27.52(4.08)$ & $26.21(4.04)$ & 0.26 \\
Period of gestation in weeks - mean (SD) & $34.43(2.52)$ & $32.5(5)$ & 0.008 \\
Primiparous (\%) & $77.87(88 / 113)$ & $64.28(9 / 14)$ & 0.317 \\
Identified source of infection (\%) & $15.04(17 / 113)$ & $7.14(1 / 14)$ & 0.690 \\
Hypertension/preeclamsia (\%) & $7.96(9 / 113)$ & $7.14(1 / 14)$ & 1.00 \\
Gestational diabetes mellitus (\%) & $11.50(13 / 113)$ & 0 & - \\
History of PROM (\%) & $2.65(3 / 113)$ & 0 & - \\
Infertility conception (\%) & $(7 / 113)$ & $(1 / 14)$ & 1.00 \\
Previous cesarean delivery (\%) & $(4 / 113)$ & $(2 / 14)$ & 0.133 \\
Other risk factors & & & - \\
Polyhydramnios & $2 / 113$ & $1 / 14$ & - \\
Multiple gestation & $9 / 113$ & 0 & - \\
Fibroid complicating pregnancy & $1-113$ & $1 / 14$ & - \\
Mullerian anomaly & 0 & $1 / 14$ & \\
\hline
\end{tabular}

\section{(B) Differential count and clinical outcome}

ROC curves were also plotted with all the components of differential leukocyte count (neutrophils, lymphocytes, monocytes and eosinophils). Figure 2 displays ROC curves describing the performance of various components of leukocyte count. ROC curve for neutrophils had an AUC of 0.801 . Neutrophil count of $80.35 \%$ gave the best combination of sensitivity $(85.7 \%)$ and specificity $(67.3 \%)$. AUC for lymphocytes, monocytes, eosinophils, basophils was 0.155 , $0.342,0.504$ and 0.481 respectively, all under 0.50 denoting just a random performance, which can not be utilized for clinical benefit.

\section{(C) Prematurity was the main cause of neonatal death} not infection

Among the 127 cases included in our study there was no case of IUD on expectant management, though there were 4 neonatal deaths. Notably three of these neonates were extremely premature, born around 27 to 28 weeks period of gestation. The fourth one though was born at 36 weeks period of gestation, however succumbed due to diaphragmatic hernia, on third postnatal day.

\section{Discussion}

Present study shows that a leukocyte count of $15,850 / \mathrm{mm}^{3}$, at the time of admission in a case of
PPROM can predict clinical outcome with a sensitivity of $85.7 \%$ and specificity of $87.6 \%$.

It is a well recognized fact that underlying infection is the cause of most of the cases of preterm labor and PPROM. To diagnose this infection at subclinical stages with the help of various markers (leukocyte count, CRP, ESR, IL-6) has been an area of interest in various studies ${ }^{7-9}$. Theoretically these tests should be able to identify the fetus in the early stages of an infectious process, before the full clinical manifestations of chorioamnionitis develops. The clinical implication of this is to prognosticate the final outcome for the mother and the newborn.

White blood cell counts are currently suggested by many, as a means of identifying infection before it becomes clinically obvious. In patients with PPROM, sensitivities as high as $81 \%$ have been reported in the literature ${ }^{9}$ Yoon et al ${ }^{11}$ evaluated leukocyte counts over $13,000 / \mathrm{mm}^{3}$ and correlated them with positive amniotic fluid cultures. They found a sensitivity of only $32 \%$ with a positive predictive value of $40 \%$ suggesting that it is of no clinical significance. The fact to be considered is leukocytosis is a recognized physiological event with pregnancy progression. Upper limit of normalcy however has not been addressed in much detail. Samuel Lurie, Einam Rahamim et al recently published their study with elaborate analysis of leukocyte count in all the three trimesters of pregnancy. After studying the pattern of 
leukocyte count in 1749 pregnancies the authors suggested that 99 th percentile should be used in defining the upper non-pathological limit for leukocyte count and leukocytes differential in pregnancy. The 99th percentile for total leukocyte count on an average was found to be $14,600 / \mu$ in which neutrophils accounted for $82.3 \%{ }^{12}$. The cut off obtained in the study conducted by Yoon et al by ROC analysis was $13,000 / \mathrm{mm}^{3}$, which is well within the limit of normalacy as evident by Samuel Lurie study. Comparing these data together it would be a better idea to correlate leukocyte count with clinical outcome rather with positive amniotic fluid cultures only.

Present study is the first study in the literature to find out a cut off for total leukocyte count to predict the outcome in pregnancies complicated with PPROM. It also emphasizes that the evaluation of differential count is of not much role to prognosticate these cases. One of the limitations of this study was the small number (14 out of 127) of subjects in the adverse outcome group (Score $\geq 8$ ). As all these cases were managed in a reputed tertiary care centre with strict observation of a team of expert gynecologists, followed by expert neonatologist with all efforts to minimize complications. Due to the same reason of rarity of adverse outcome in a comparatively long study period, these results were not validated in this study. Future studies are required to validate these results in other clinical settings.

\section{Conclusion}

Leukocyte count of $15,850 / \mathrm{mm}^{3}$, at the time of admission was found to be able to predict the clinical outcome with a sensitivity of $85.7 \%$ and specificity of $87.6 \%$, in a case of PPROM. Whereas the individual components of differential leukocyte counts were found to be not of much help in this regard.

\section{Acknowledgements}

We acknowledge Professor Lavanya Rai for permitting us to include their patients in the study; and Dr Asha Shanbag for her help in data collection.

\section{References}

1. Mercer BM, Goldenberg RL, Meis PJ, Moawad AH, Shellhaas C, Das A, et al. The Preterm Prediction Study: prediction of preterm premature rupture of membranes through clinical findings and ancillary testing, The National Institute of Child Health and Human Development Maternal-Fetal Medicine Units Network. Am J Obstet Gynecol 2000; 183(3): 738-45.

2. Merenstein GB, Weisman LE. Premature rupture of the membranes: neonatal consequences. Semin Perinatol 1996; 20(5): 375-80.

3. Furman B, Shoham-Vardi I, Bashiri A, Erez O, Mazor M. Clinical significance and outcome of preterm prelabor rupture of membranes: population-based study. Eur J Obstet Gynecol Reprod Biol 2000; 92(2): 209-16.

4. Brian M, Mercer BA. Is there a role for tocolytic therapy during conservative management of preterm premature rupture of the membranes? Clinical Obstetrics and Gynecology 2007; 50: 487-96.

5. Caughey AB, Robinson JN, Norwitz ER. Contemporary diagnosis and management of preterm premature rupture of membranes. Rev Obstet Gynecol 2008; 1(1): 11-22.

6. Roberts D, Dalziel S. Antenatal corticosteroids for accelerating fetal lung maturation for women at risk of preterm birth. Cochrane Database Syst Rev 2006; 3: CD004454. Edited, published in Issue 9, 2010.

7. Shim SS, Romero R, Jun JK, Moon KC, Kim G, Yoon BH. Creactive protein concentration in vaginal fluid as a marker for intra-amniotic inflammation/infection in preterm premature rupture of membranes. J Matern Fetal Neonatal Med 2005; 18(6): 417-22.

8. Asrat T. Intra-amniotic infection in patients with preterm prelabor rupture of membranes: pathophysiology, detection, and management. Clinics in Perinatology 2001; 28: 735-51.

9. Greig PC. The diagnosis of intrauterine infection in women with preterm premature rupture of the membranes (PPROM). Clin Obstet Gynecol 1998; 41: 849,.

10. Van de Laar R, Van der Ham DP, Oei SG, Willekes C, Weiner CP, Mol BW. Accuracy of C-reactive protein determination in predicting chorioamnionitis and neonatal infection in pregnant women with premature rupture of membranes: a systematic review. European J of Obstet Gynecol Reproductive Biol 2009; 147(2): 124-9.

11. Yoon BH, Jun JK, Park KH, et al. Serum C-reactive protein, white blood cell count, and amniotic fluid white blood cell count in women with preterm premature rupture of membranes. Obstet Gynecol 1996; 88: 1034.

12. Lurie S, Rahamim E, Piper I, Golan A, Sadan O. Total and differential leukocyte counts percentiles in normal pregnancy. European Journal of Obstetrics \& Gynecology and Reproductive Biology 2008; 136: 16-9. 